\title{
Reinforcement of schedule-induced drinking in rats by lick-contingent shortening of food delivery
}

\author{
Beatriz Álvarez ${ }^{1}$ Javier Íbias ${ }^{1}$ • Ricardo Pellón ${ }^{1,2}$
}

Published online: 8 April 2016

(C) Psychonomic Society, Inc. 2016

\begin{abstract}
Schedule-induced drinking has been a theoretical question of concern ever since it was first described more than 50 years ago. It has been classified as adjunctive behavior; that is, behavior that is induced by an incentive but not reinforced by it. Nevertheless, some authors have argued against this view, claiming that adjunctive drinking is actually a type of operant behavior. If this were true, schedule-induced drinking should be controlled by its consequences, which is the major definition of an operant. The present study tested this hypothesis. In a first experimental phase, a single pellet of food was delivered at regular 90-s intervals, but the interfood interval could be shortened depending on the rat's licking. The degree of contingency between licking the bottle spout and hastening the delivery of the food pellet was $100 \%, 50 \%$, and $0 \%$ for 3 separate groups of animals. Rats that could shorten the interval (100\% and $50 \%$ contingency) drank at a higher rate than those that could not $(0 \%)$, and the level of acquisition was positively related to the degree of contingency. In a second phase of the experiment, all groups were exposed to a $100 \%$ contingency, which resulted in all rats developing high levels of schedule-induced drinking. Licking is enhanced if it hastens reinforcement, and can do so at delay characteristics of those present in studies of schedule-induced drinking, thus supporting the view that adjunctive behavior is an operant.
\end{abstract}

Ricardo Pellón

rpellon@psi.uned.es

1 Animal Behavior Laboratories, Departamento de Psicología Básica I, Facultad de Psicología, Universidad Nacional de Educación a Distancia, Madrid, Spain

2 Departamento de Psicología Básica I, Facultad de Psicología, Universidad Nacional de Educación a Distancia, C/ Juan del Rosal 10, Ciudad Universitaria, 28040 Madrid, Spain
Keywords Schedule-induced drinking · Adjunctive vs. operant behavior $\cdot$ Lick-food contingency $\cdot$ Rats

Schedule-induced drinking was first described by Falk when he was running an experiment with food-deprived rats on bar pressing under a variable-interval 1-minute food reinforcement schedule in which the animals had continuous access to a bottle of water (Falk, 1961). He observed that rats, despite not being water deprived, consumed large quantities of water (polydipsia) by following the regular pattern of accumulating small quantities after the delivery of each food pellet. As deprivation of food normally leads to a decrease in water intake (e.g., Bolles, 1961), and drinking was not explicitly reinforced, this excessive consumption of water was described by Falk as "absurd" (Falk, 1971, p. 577). The main characteristics of schedule-induced drinking are therefore that (a) it takes place after the food pellet has been delivered and (b) in an exaggerated manner. This large consumption of water occurs under an intermittent food schedule and with no apparent reason, as it does not appear to be reinforced during training.

\section{Characterization of schedule-induced drinking as an adjunctive behavior}

One of the approaches to explain schedule-induced drinking is to consider this as a phenomenon belonging to a separate category of behavior: adjunctive (Falk, 1971; see a critical analysis by Wetherington, 1982). According to this point of view, adjunctive drinking is induced by the delivery of periodic food but is not reinforced by it (see Baum, 2012, for a recent treatment of induction).

Along with drinking, there are other behaviors that can be excessively induced by intermittent food schedules or other 
intermittent programmed commodities. Some of those behaviors are pica, aggression, escape, wheel running, and air licking (Falk, 1971; Pellón, 1990). All of them share certain characteristics regarding their controlling variables, and, therefore, according to Falk, they should be considered as a group of behavioral phenomena.

Adjunctive behaviors broadly share the following characteristics: (a) They are strongly determined by the degree of deprivation of the intermittently programmed commodity because for an exaggerated adjunctive behavior to be developed, a relatively moderate level of deprivation of that commodity must be ensured (Falk, 1969, for studies on food deprivation and schedule-induced drinking). (b) Their acquisition is determined not solely by the degree of commodity deprivation but by the rate at which it is presented (Falk, 1966b; Flory, 1971, for studies on food frequency and schedule-induced drinking), and not by a contingency between any given response and reinforcement (Flores \& Pellón, 1995, for no differences between interval and time schedules in the generation of drinking). (c) They show a regular pattern and are displayed excessively compared to the baseline rate of the behavior occurrence when is not under an intermittent schedule (Roper, 1981). (d) They take place immediately after the commodity has been delivered, in the postreinforcement period and not in the prereinforcement period, like normal operants (but see, e.g., López-Crespo, Rodríguez, Pellón, \& Flores, 2004). (e) They develop and can be substituted for another adjunctive behavior, depending on the constraints offered by the experimental environment (e.g., Knutson \& Schrader, 1975). (f) Once developed, they can become reinforcing behaviors in their own right, thus being capable of sustaining another behavior (e.g., Falk, 1966a). (g) There is an optimal proportion between the length of the interreinforcement interval and the quantity delivered of the commodity that favors the development of adjunctive behavior. In the case of schedule-induced drinking, Falk (1967) showed that it was not the duration of the interfood intervals on its own, nor the amount of food given in each trial, but the proportion maintained between both of them that appears to matter. For a more extended consideration of the defining characteristics of adjunctive behavior, see Falk (1971).

\section{Characterization of schedule-induced drinking as an operant behavior}

An alternative view of schedule-induced drinking is to consider it as an operant behavior and not as a separate class of behavior. An operant is defined as a behavior that takes place because its consequences increase the probability of its occurrence (e.g., Mazur, 1998, p. 136), which, applied to schedule-induced drinking, will mean that it is developed because the delivery of food pellets actually reinforces drinking (see Clark, 1962, for an early account in these terms).

Similarities between adjunctive drinking and operant behavior have been emphasized before (Íbias, Pellón, \& Sanabria, 2015; Pellón, 2004). These similarities are related to acquisition, levels of motivation, reinforceability, temporal control, and sensitivity to contingent reinforcement and are summarized below (for a more detailed review and theoretical consideration, see Killeen \& Pellón, 2013).

As well as operants, (a) schedule-induced drinking covaries with food motivation (i.e., level of food deprivation, quantity and quality of food delivered) and not with water motivation, this having been shown in studies of acquisition (Falk, 1967; Lamas \& Pellón, 1997) and punishment of schedule-induced drinking (Lamas \& Pellón, 1995), thus setting the ground to the suggestion that adjunctive drinking is reinforced by food but not by water. (b) In terms of acquisition, it has been shown that those behaviors that are subject to reinforcement can be developed as adjuncts, and that if they do not respond to contingent reinforcement, they do not occur as adjuncts. Moreover, (c) adjunctive behavior (in particular drinking) is acquired at the same rate as operant behavior maintained by delayed reinforcement (Killeen \& Pellón, 2013), showing, in this case, similar temporal patterns, and thus (d) the fact that adjunctive drinking is normally a postpellet phenomenon, whereas operants are usually prepellet, does not preclude adjunctive behavior from being considered an operant (Killeen \& Pellón, 2013). Finally, (e) schedule-induced drinking is sensitive to the contingencies established between the behavior of licking and the delivery of food (e.g., Pellón \& PérezPadilla, 2013).

\section{Are adjuncts operants?}

The above-mentioned similarities between adjuncts and operants could be indicating that adjunctive drinking is actually a type of operant behavior and that it does not constitute a separate class of behavior, as first suggested by Falk (1971). Adjunctive behavior could actually be a kind of operant behavior that is developed under a delayed-reinforcement schedule, as has been recently formally proposed by Killeen and Pellón (2013). The aim of the present work was to further test this hypothesis.

Pellón and Blackman (1987) ran a series of experiments in which they showed how schedule-induced drinking could be suppressed by lick-contingent delays in food presentation. Each lick given by a rat initiated a delay of 10 seconds in the delivery of the next food pellet. Even though the behavior of drinking was not completely suppressed, the establishment of this contingency led to lower rates of drinking regardless of whether or not the delays were signaled. These results were later replicated (Lamas \& Pellón, 1995; Pellón \& Castilla, 
2000) and have been shown to be very similar to the results normally found when a well-established operant behavior is punished. It has also been seen that resistance to punishment is related to the deprivation levels in operant behavior, just as it is in schedule-induced drinking (Lamas \& Pellón, 1995).

All the above-mentioned studies tested the reinforceability of schedule-induced drinking under a punishment schedule. The findings that schedule-induced drinking can be modulated by contingent reinforcement do not imply that the behavior developed as a consequence of contingent reinforcement in the first place. In other words, schedule-induced drinking can be initiated by nonoperant processes but once acquired becomes sensitive to differential reinforcement. To our knowledge, there are no studies that have tried to address the question of whether or not schedule-induced drinking can be acquired in an operant fashion. Solving this question could help to better understand whether adjuncts and operants are the same. For this reason, the present work established a positive contingency between the behavior of drinking and the delivery of food pellets. In the first phase of the study, a contingency between licking and the shortening of the interfood pellet trial was manipulated for three different groups. For one group, a $100 \%$ contingency was established; thus, rats belonging to this group were able to shorten the length of all interfood intervals. For a second group, the contingency established was $0 \%$, and for a third group, a $50 \%$ contingency was used. In a second phase of the study, a $100 \%$ contingency was established for all groups. By means of this design, a better understanding of schedule-induced drinking (and by extension, of adjunctive behavior) will be achieved. If the acquisition of schedule-induced drinking does not respond to contingency rules, either no difference should be found between groups or differences should relate to experienced values of interfood intervals. If, on the other hand, schedule-induced drinking functions as an operant, the largest amount of licking (and water intake) should be shown by rats under the $100 \%$ contingency, followed by rats under the $50 \%$ condition, and rats with $0 \%$ contingency should show the least licking.

\section{Method}

\section{Subjects}

Subjects were 24 young male Wistar rats from Charles River Laboratories (Lyon, France). At their arrival, they were 10 weeks old and experimentally naïve. They were kept individually in transparent Plexiglas cages measuring $18 \times 32.5 \times$ $20.5 \mathrm{~cm}$ in an environmentally controlled room $\left(22^{\circ} \mathrm{C}\right.$ and $60 \%$ relative humidity). A 12-hour light-dark cycle was maintained (lights on at 8 a.m.). Animals' weights were gradually reduced by controlled feeding to $85 \%$ of their individual freefeeding weights and were kept at those weights throughout the experiment. At the start of the experiment, rats were in their 16th week of life and had an average weight of 343 grams (range: $310-378$ grams). Animal care procedures were in accordance with the European Union Council Directive 2010/63 and the Spanish Royal Decree 53/2013.

\section{Apparatus}

Eight Letica LI-836 conditioning chambers, $29 \times 24.5 \times$ $35.5 \mathrm{~cm}$, enclosed in sound-attenuating housing, were used. They were equipped with their own ventilation and a small observation window at the front. The front panel of each conditioning chamber was made of aluminum, the left wall of transparent Plexiglas, and the remaining walls of black Plexiglas. On the exterior right-hand wall of the chamber, a water bottle was fitted, with a spout to which the rat had access from the interior of the chamber, through a $3.2-\mathrm{cm} \times 3.9-\mathrm{cm}$ aperture in the wall, situated $20 \mathrm{~cm}$ from the front panel and $7 \mathrm{~cm}$ from the floor. The spout was placed $2 \mathrm{~cm}$ toward the interior of the aperture to allow for licks rather than continuous drinking. Contact between the animal's tongue and the metal spout completed the electric circuit between the 16-bar metal grid floor and the water-bottle spout. Licks were recorded using a MED-PC application under a Windows environment. Forty-five-mg food pellets were dispensed (Bio-Serv, Frenchtown, NJ, USA) in an aperture in the front wall of the chamber, situated $3.7 \mathrm{~cm}$ from the floor, between the two panel levers, which were retracted throughout the experiment. The chambers were lit by two 3-W lamps situated on the front panel at either side of the food hopper and by an indirect $25-\mathrm{W}$ light fitted to the interior of the sound-attenuating housing that insulated each chamber. A fan that produced an ambient noise of approximately $60 \mathrm{~dB}$ in each chamber masked exterior noise.

\section{Procedure}

Rats were divided into three different groups $(n=8)$ : master, yoked control, and $50 \%$ contingency. The experiment consisted of two phases, with sessions conducted 5 days per week, from Mondays to Fridays, at about the same time every day. Before each session, the water bottles were filled with $100 \mathrm{ml}$ of fresh tap water and installed in the conditioning chamber. Each session began with the illumination of the houselights, which were turned off at the end of each experimental session.

In the first phase (Phase 1), 15 training sessions were run, of 30 trials each. For the master group, a fixed time (FT) 90-s schedule was used: a food pellet was delivered every $90 \mathrm{sec}-$ onds without specific response requirement. However, every 10 licks given by the rat shortened the FT value by 20 seconds, up to a maximum reduction of interfood intervals of $60 \mathrm{sec}-$ onds (as a result of the first 30 licks - 3 times 10 licks). Therefore, each interfood interval could last any time between 30 and 90 seconds, depending on whether the rat made, 
respectively, 30 or more licks, 20 to 29 licks, 10 to 19 licks, or less than 10 licks.

Each master rat had a yoked control rat, such that this yoked rat would receive the food pellet at the same time as its master but had no control over its occurrence by its own licking. Food delivery to both rats was thus affected by the master rat's licking. For the $50 \%$ contingency group, there were two types of trials. Type I trials were the same as the trials for the yoked control group (the contingency between their licking and shortening the interval was 0), and Type II trials were the same as for the master group (the contingency between licking and shortening the interval was $100 \%$ ). This way, in $50 \%$ of the trials, subjects belonging to the $50 \%$ contingency group received the food pellet when their master received it; in the other half of the trials, they were able to shorten the duration of the interval. Type I and Type II trials were randomly distributed.

In Phase 2, another 15 sessions of training were run. During this second phase, every subject, irrespective of the group, was under the schedule used for the master group during Phase 1. Each animal was now able to accelerate the delivery of the food pellet by means of its licking behavior.

The duration of the session, the length and number of the different interfood intervals, the volume of water consumption, the number of licks at the spout and the number of head entries to the magazine, were recorded for each rat in each session. Licks and magazine entries were also recorded in 1$\mathrm{s}$ bins of interfood intervals.

\section{Results}

The main results of the study are shown in Fig. 1, which represents the mean number of licks (upper panel), magazine entries (middle panel), and food pellets per minute (lower panel) given by each group of rats in the two phases of the experiment. All these data were analyzed by means of two-factor ANOVAs: a repeated-measures factor, sessions (with 15 levels), and a fixed factor, group (with 3 levels). Bonferroni adjustment was used in post hoc comparisons when necessary, effect sizes were presented as $\eta_{\mathrm{p}}{ }^{2}$, and the level of significance was established at a minimum $p<.05$. Analyses were executed with SPSS 19.0, and were performed independently for each experimental phase and separately for licks, magazine entries, and food frequency. Experimental phases were treated separately because it was intended to evaluate the influence of the degree of lick-food contingency (the critical variable under investigation) on the acquisition of schedule-induced drinking, without confounding those data with the ones obtained in a second experimental phase that equalized the degree of contingency among all groups with the purpose of studying if the differences obtained in the first phase would be abolished by this significant change in the experimental arrangement.

With regard to licks, during Phase 1 (see Fig. 1, left-hand side of the panels), master rats licked more than the $50 \%$ contingency and yoked control animals, the $50 \%$ contingency licking rate was higher than that of the yoked control, and the amount of licks given increased as sessions continued for all the three groups (albeit not to a great degree in the yoked condition). These general findings were confirmed by the statistical analysis. A group effect was found, $F(2,21)=7.15, p<$ $.01, \eta_{\mathrm{p}}{ }^{2}=0.41$ : Master animals showed a larger number of licks compared to the yoked and $50 \%$ contingency rats $(p<$ .01 and $p<.04$, respectively). An effect of sessions was also found, $F(14,294)=16.11, p<.01, \eta_{\mathrm{p}}{ }^{2}=0.43$, reflecting that average licks increased along Phase 1. Finally, an interaction Group $\times$ Sessions was obtained, $F(28,294)=2.54, p<.01$, $\eta_{\mathrm{p}}{ }^{2}=0.20$, revealing that master rats showed higher levels of response than did yoked control rats from Session 2 throughout the rest of the training $(p<.05)$, and that the master group also differed from the $50 \%$ contingency group but only from Session 2 to Session $8(p<.05)$. The master group showed higher levels of response than the other two groups throughout the experiment, but from Session 9 onwards, the $50 \%$ contingency group did not show statistical differences with respect to the master and yoked control groups, reaching levels of licking that were in between those of master and yoked control rats. The sessions in which the groups differed are detailed in the left upper panel of Fig. 1.

In Phase 2 (right-hand side of the panels of Fig. 1), the mean number of licks given by the yoked control and $50 \%$ contingency groups increased over the sessions, reaching final levels of licking that were comparable among all groups (albeit to a lower level in the yoked control group). Statistically, only an effect of sessions was found, $F(14,294)=8.00, p<.01, \eta_{\mathrm{p}}{ }^{2}=0.28$.

Results of licking were accompanied by data on water consumption. At the end of Phase 1, the groups of rats drank as follows (mean $\mathrm{mL} \pm S D$ ): master $8.88 \pm 3.15,50 \%$ contingency $8.50 \pm 3.94$, and yoked $6.76 \pm 2.40$; and at the end of Phase 2, as follows: master $10.47 \pm 3.28,50 \%$ contingency $10.00 \pm 3.81$, and yoked $9.27 \pm 2.70$.

Entries to the magazine were also analyzed and are represented in the middle panel of Fig. 1 as a function of groups and sessions. As can be seen, during Phase 1 (left-hand side), the $50 \%$ contingency was the group with the highest level of magazine entries throughout all sessions, followed by the yoked and master groups, in that order. Differences due to group were found, $F(2,21)=8.02, p<.01, \eta_{\mathrm{p}}^{2}=0.43$; the $50 \%$ contingency group showed a significantly higher number of magazine entries in contrast with master rats $(p<.01)$, but no differences between the $50 \%$ contingency and yoked control groups were found. Between the master and yoked groups, no statistical differences were found despite the 
Fig. 1 Mean number of licks, magazine entries, and food pellets per minute by the three groups of rats for each session of the two phases of the experiment. Note. \# $=$ significant differences between master and yoked groups. \#\# = significant differences between master and $50 \%$ contingency groups. Basal reinforcement rate $(=0.67)$ depicts the number of food pellets per minute delivered by the FT 90 -s schedule when none of the intervals was shortened. Error bars denote SEM
PHASE I

PHASE II
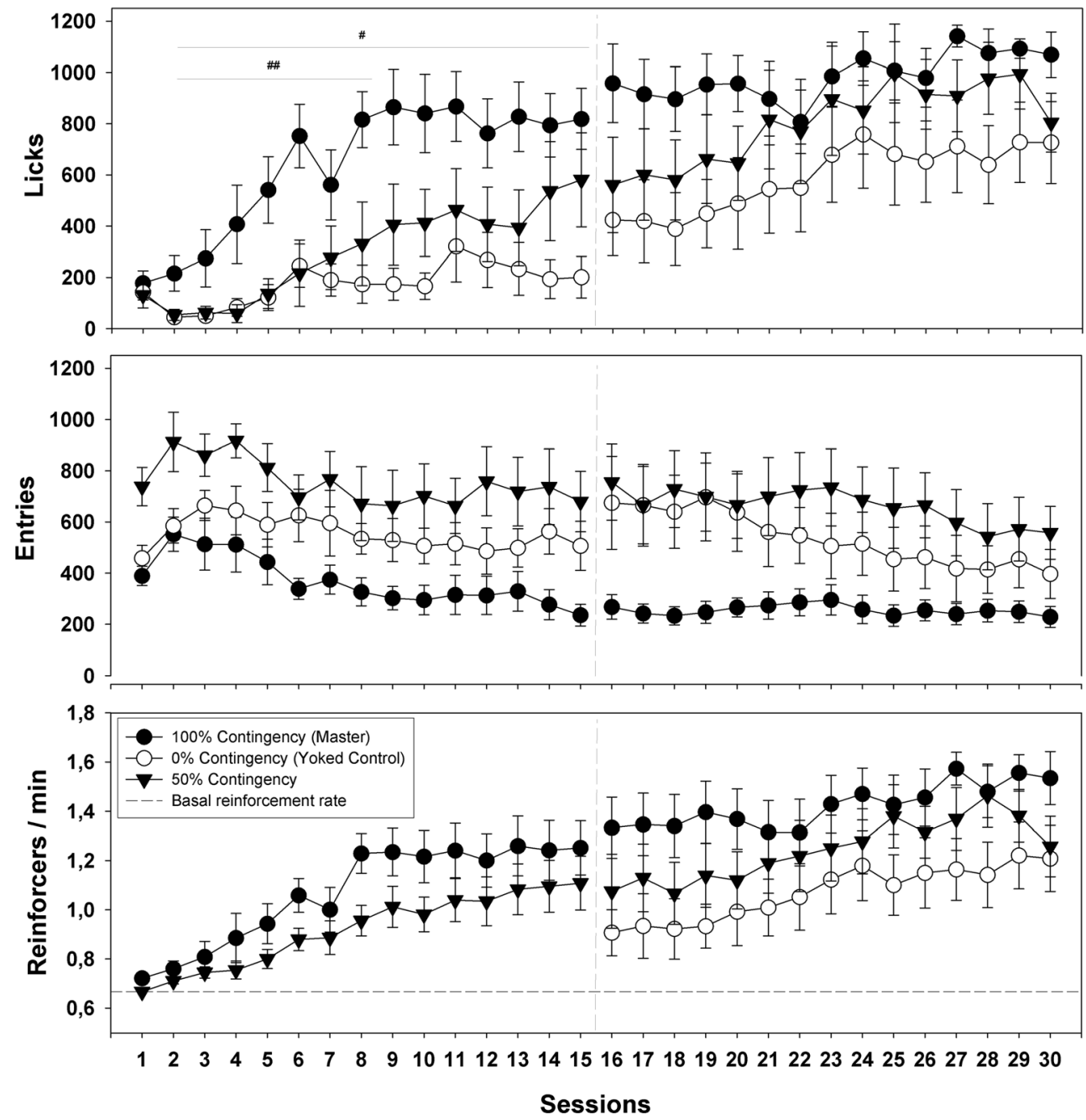

tendency of master rats to make fewer head entries from Session 3 till the end of training. No differences were found in terms of sessions, even when analyzing each group independently. Thus, each group showed similar means of magazine entries throughout the sessions of Phase 1.

In Phase 2 (right-hand side of the middle panel of Fig. 1), the same tendency in the results was observed as in Phase 1. There was only a group effect, $F(2,21)=4.00, p=.03, \eta_{\mathrm{p}}{ }^{2}=$ 0.28 . The $50 \%$ contingency group had the highest level of response and did not show statistical differences with yoked control animals, whereas master rats had the lowest levels of magazine entries, as in Phase $1(p<.04$ for the difference between the master and $50 \%$ contingency groups). Despite the absence of statistical differences, as schedule-induced drinking was being developed by yoked control animals during Phase 2 (see Fig. 1, upper panel), a tendency to lower the amount of magazine entries was observed. At the beginning of Phase 2, yoked control animals slightly increased their response level and then lowered it as sessions went by. This result is comparable to that found in Phase 1 in the master group during the first five sessions.

Rate of food delivery increased as sessions progressed during both Phase $1, F(14,196)=20.44, p<.01, \eta_{\mathrm{p}}{ }^{2}=0.59$, and Phase $2, F(14,294)=8.85, p<.000, \eta_{\mathrm{p}}{ }^{2}=0.30$, as can be seen in the left- and right-hand bottom panels of Fig. 1, respectively. Neither a group effect nor a Group $\times$ Session interaction was obtained in either phase of the study: Phase 1: $F(1,14)=$ $2.89, p<.11, \eta_{\mathrm{p}}{ }^{2}=0.17$, and $F(14,196)=0.73, p<.74, \eta_{\mathrm{p}}{ }^{2}=$ 0.05 , respectively; Phase 2: $F(2,21)=2.58, p<.10, \eta_{\mathrm{p}}^{2}=$ 0.20 , and $F(28,294)=0.72, p<.86, \eta_{\mathrm{p}}{ }^{2}=0.06$, respectively. Food frequency increased in correspondence with increases in licking, and this occurred during Phase 1 both for master rats (and their accompanying yoked controls) and the $50 \%$ contingency rats, and during Phase 2 for the yoked and $50 \%$ contingency groups, thus contributing to the overall increase in food rate that was obtained as sessions progressed during Phase 2. Having shown no statistical difference between groups, the possibility exists that the general higher reinforcer 
rates seen for master rats along Phase 1 contributed to their elevated licking. This possibility is unlikely, however, because if a shorter interfood interval had caused elevated licking in the $100 \%$ condition relative to the $50 \%$ condition, then it should have been observed the same in the yoked control group because the $100 \%$ and $0 \%$ conditions had identical interval durations (see a more extended treatment of this in the Discussion section).

Figure 2 shows the number of interfood intervals of different lengths that were experienced by master and yoked control rats (100\% and $0 \%$ contingencies) versus the $50 \%$ contingency animals, throughout the entire sessions of Phase 1. A statistical analysis was applied similar to those performed on the data of Fig. 1, but with a length factor (with 4 levels) and a group factor (with 2 levels). It can be seen that most of the interfood intervals experienced were either long (80-90 seconds) or short (30-40 seconds), and that much fewer intermediate interfood intervals occurred. A length effect was obtained, $F(3,42)=20.19, p<.01, \eta_{\mathrm{p}}{ }^{2}=0.59$. There was a tendency for the $50 \%$ contingency to experience longer interfood intervals, while the opposite occurred for the $100 \%$ and $0 \%$ groups (in line with overall reinforcement rates reported in Fig. 1). However, neither a group effect nor a Group $\times$ Length interaction, $F(3,42)=2.30, p<.09, \eta_{\mathrm{p}}{ }^{2}=0.14$, were obtained.

A further analysis of the results was conducted in terms of temporal distributions of licks and magazine entries within interfood intervals. Figure 3 depicts mean total licks given every 1 -s bin during the entire interfood intervals that composed Sessions 1, 2, 5, 8, 11 and 15, both of Phase 1 (left panels of Fig. 3) and Phase 2 (right panels of Fig. 3; here numbered 16, 17, 20, 23, 26 and 30). As trials did not have the same duration, because their duration was dependent on the behavior of the rats (intervals could last any time between 30 and 90 seconds), a ratio was calculated in order to compare the amount of licks given during each trial and session. The ratio obtained is the mean number of licks given by each rat of each group at each second of each interval. As can be seen in Fig. 3, master rats developed schedule-induced drinking quite

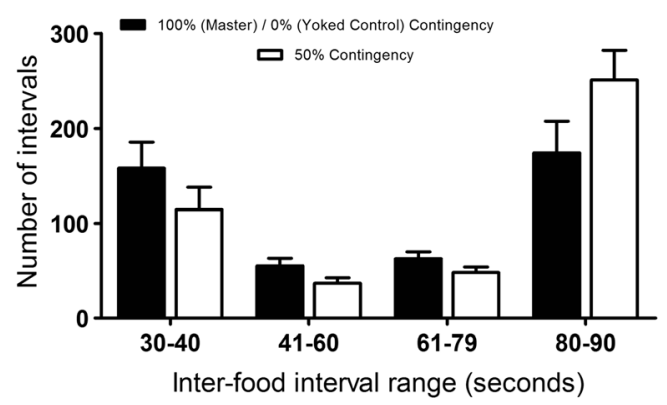

Fig. 2 Mean number of interfood intervals of different lengths throughout the entire sessions of Phase 1. Interfood intervals were grouped in ranges of 30-40, 41-60, 61-79, and 80-90 seconds. Error bars denote $S E M$ quickly in the first phase of the experiment (by Session 5), and licking occurred at the beginning of the interfood intervals (clearly seen in Sessions 8, 11, and 15); that is, a few seconds after the food pellet had been delivered. On average, the behavior of drinking lingered during the first 30 seconds of the interval and then vanished.

Until Session 8 , the $50 \%$ contingency rats did not begin licking, and until Session 11 they did not do so significantly, and always a few seconds later in relation to the delivery of the food pellet compared to master rats. By the 15 th session, they had adjusted their licking curve to that of the master subjects. A second imbalance was observed during the two first sessions of Phase 2, but by Sessions 20 to 23, the rats had again readjusted their licking curve to the masters' distribution. Yoked control animals took longer to develop licking and, even though there were no significant differences with the other two groups in the second phase of the experiment, the distribution of licks was considerably lower than that of both the master and $50 \%$ contingency groups. Previous experience, where no contingency was established between licking and the delivery of food pellets, seems to have impaired learning about this new condition, as is clearly depicted in Fig. 3. Nevertheless, the distribution of licking was similar to that of the master and $50 \%$ contingency groups; licks were concentrated during the first 30 seconds of the interval (which is marked by a dashed vertical line on the $x$-axis).

In terms of entries to the magazine, Fig. 4 depicts how they were distributed along the interfood intervals for Session 1 of Phase 1, and Sessions 16 and 30 of Phase 2. As can be seen, most magazine entries of the master and yoked control groups took place at the beginning of the interval (Sessions 16 and 30 of Phase 2), just when the food pellet was being delivered. For the $50 \%$ contingency group, there was a second peak of magazine entries at the end of the 30 first seconds of the interval. The other two groups also showed an increase of magazine entries as the interfood intervals approached $30 \mathrm{sec}$ onds but to a lower extent than the $50 \%$ contingency rats, which was the main determinant of their general higher level of magazine entries (see Fig. 1, middle panel). This biphasic pattern of magazine entries developed with training, as no evidence of such organization was seen during the first experimental session, where magazine entries occurred equally along the session and for all groups of rats (upper panel).

\section{Discussion}

The present investigation aimed to test whether scheduleinduced drinking can be considered an operant behavior - an issue that has remained debatable ever since the phenomenon was first reported (see Wetherington, 1982). As operants are defined by their consequences, a way to test this hypothesis would be to establish a contingency between the behavior of 

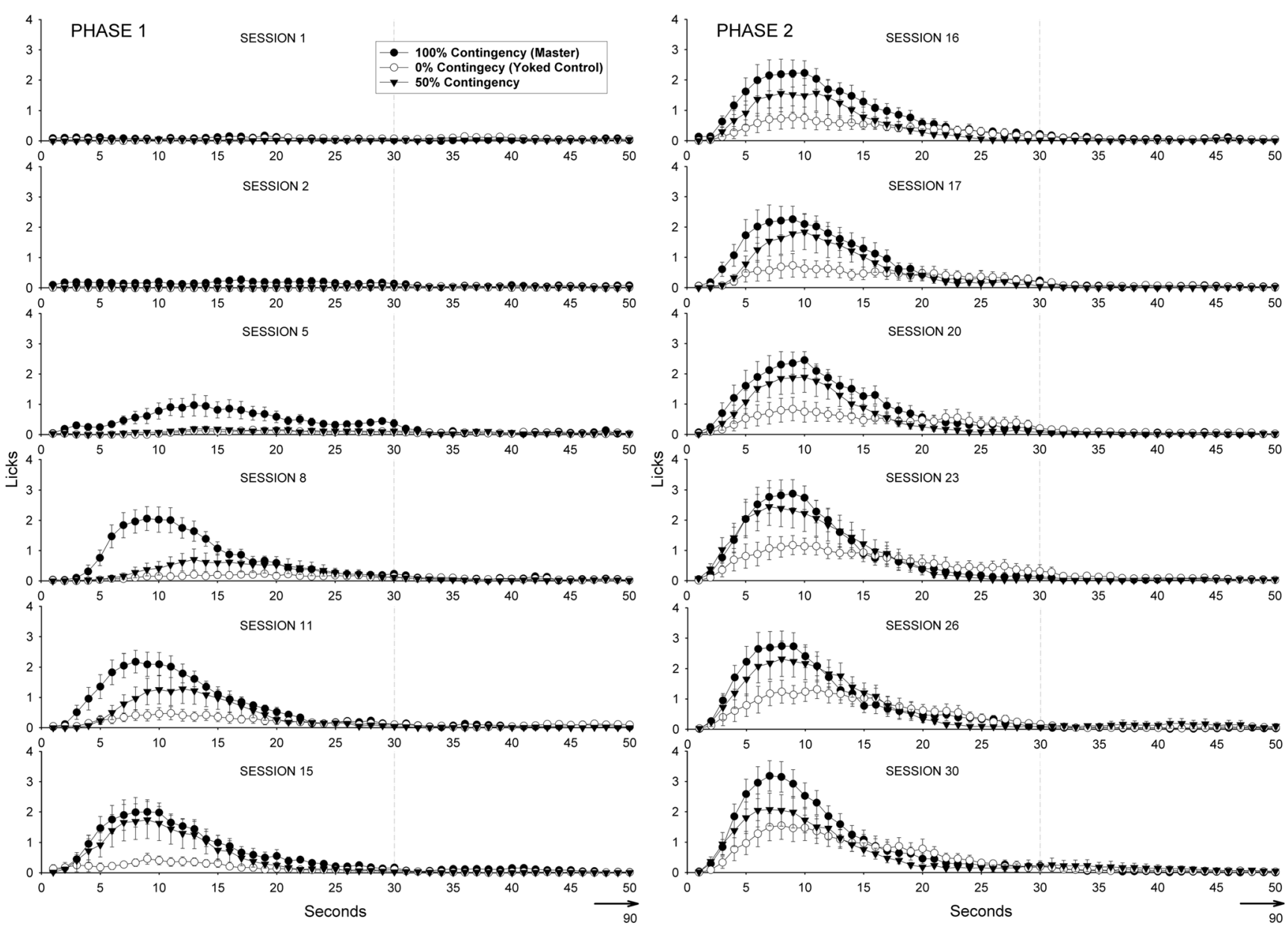

Fig. 3 Distribution of licks of the three groups of rats along interfood intervals of Sessions 1, 2, 5, 8, 11 and 15 of Phase 1 (left-side panels, from top to bottom), and Sessions 16, 17, 20, 23, 26 and 30 of Phase 2 (right-side panels, from top to bottom). Error bars denote SEM

Fig. 4 Distribution of magazine entries of the three groups of rats along the interfood intervals of Session 1 of Phase 1 (upper panel) and Sessions 16 and 30 of Phase 2 (lower two panels). Error bars denote $S E M$

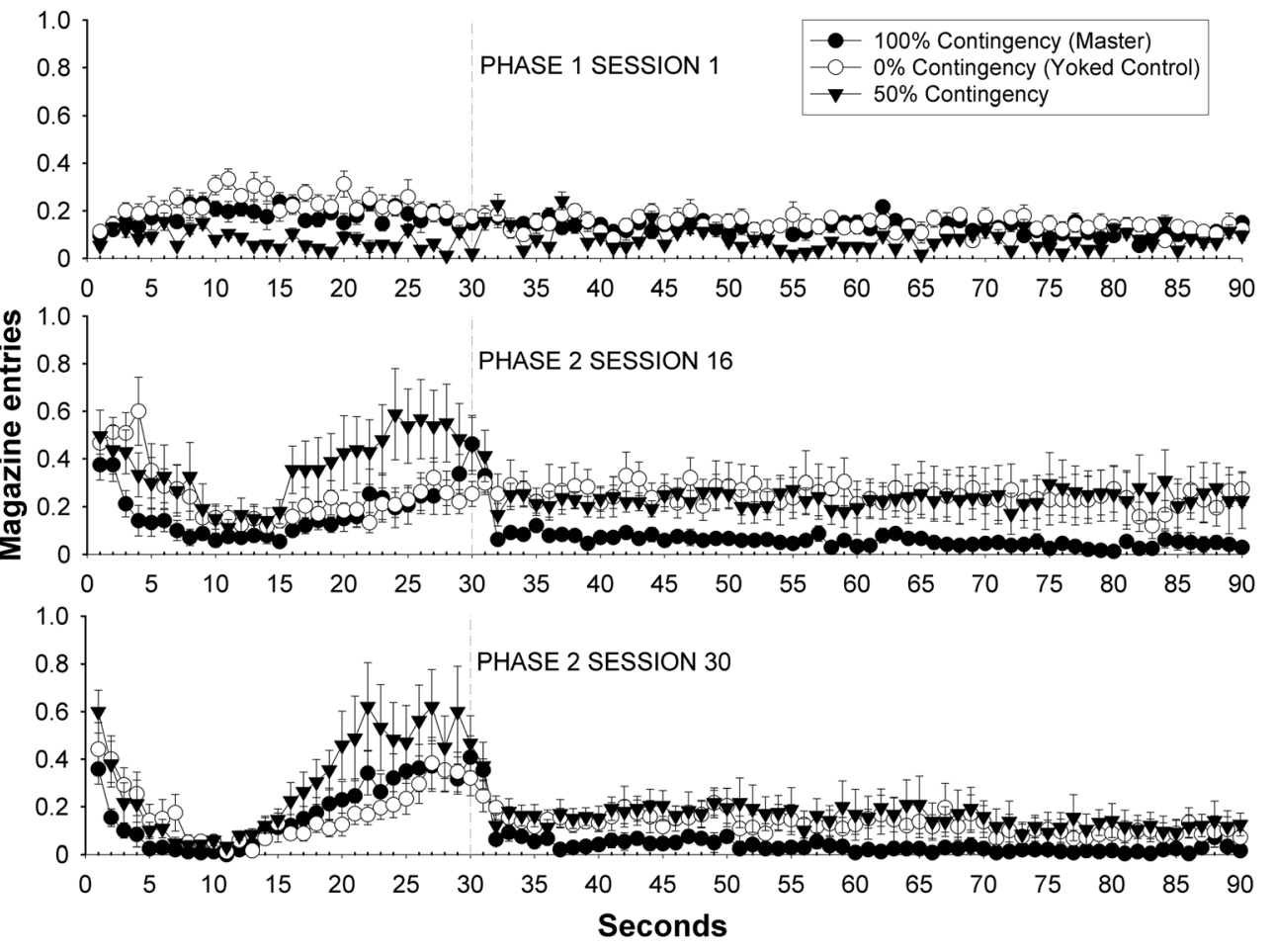


licking a waterspout and the delivery of food pellets. This contingency has previously been employed in several experiments in which the behavior of drinking was punished by a delay in the delivery of food (Lamas \& Pellón, 1995; Pellón \& Blackman, 1987; Pellón \& Castilla, 2000), thus lengthening the interfood interval. Those studies concluded that adjunctive drinking was subject to punishment but left unanswered the question of whether it was developed by a contingent food schedule (see Patterson \& Boakes, 2012). The present research was specifically designed to test this issue.

In the two-phase experiment conducted, animals belonging to the master group were able to shorten the intervals of food presentation by giving licks to the spout. This contingency between licking and the shortening of the intervals was lowered to $50 \%$ for a second group, whereas a third group was not able to shorten the intervals at all (as it was yoked to the master group). In Phase 1 (Fig. 1, upper panel) the acquisition of schedule-induced drinking for the master animals was facilitated by the established possibility of shortening the length of the interfood intervals. On the contrary, the yoked control and $50 \%$ contingency groups showed a delay in terms of acquisition in comparison to the master group: the $50 \%$ contingency group developed schedule-induced drinking but more slowly, and acquisition was almost impaired in the yoked control group. In the second phase of the experiment, all subjects were able, via licking, to control the duration of the interfood interval (100\% contingency), and all of them developed (or continued developing) schedule-induced drinking to a similar extent by the end of training: Master rats kept increasing their licking responses, the $50 \%$ contingency animals reached the level of schedule-induced drinking acquired by the masters, and yoked rats developed licking although not in such a strong, apparent fashion. The relative impairment of acquisition of schedule-induced drinking by yoked control rats resembles the findings in studies of learned helplessness where noncontingent food delivery retards or impedes the later acquisition of response control over food delivery (e.g., Job, 1988). Furthermore, this identifies another instance in which a prior history with little possibility to drink affects subsequent development of schedule-induced drinking (e.g., Tang, Williams, \& Falk, 1988).

Food frequency increased corresponding with increases in licks during acquisition of schedule-induced drinking (Fig. 1, lower panel), but, as there was a limit to the shortening of the interfood intervals due to licking, no statistical differences in rate of food delivery were observed among groups, which ensured a nondifferent rate of food administration so that the effect of the food contingency can be interpreted more easily. Furthermore, groups also did not differ statistically in the variety and length of interfood intervals that they experienced due to their differential licking (see Fig. 2). The small nonsignificant statistical reduction in the rate of food delivery of the $50 \%$ contingency group cannot account for the lower licking shown by these animals in comparison to the $100 \%$ master rats, because if this were the critical variable yoked control rats should have shown a high licking similar to master animals, but they did not. Also, a range of interfood intervals between 60 to 70 seconds (the approximate values for $100 \%$ vs. $50 \%$ groups at the end of the first phase of training) has never been reported to be sufficient to sustain different levels of scheduleinduced drinking (e.g., Falk, 1966b; Flory, 1971). The differences between licking corresponding to level of contingency should be attributed to the degree of control that licking exerted over shortening food delivery, which was ordered by $100 \%>50 \%>0 \%$, and not to any difference on the rate of food delivery or on how food delivery was experienced.

An interesting finding from the present data is the temporal location of drinking as it progressively developed. First, licks were located at the beginning of the interfood intervals, even at early moments in the development of the behavior, in a manner reflecting post-prandial drinking (Kissileff, 1969). However, initially, some licking was spread throughout the interfood intervals (see Fig. 3, Session 5 for master rats and Session 8 for the $50 \%$ contingency group) before licking became concentrated almost exclusively at the start of the intervals (see Session 15 for final performance), in keeping with previous reports that have shown an initial occurrence of licking late in interfood intervals and how, with training, its temporal location moves backwards to earlier portions typical of schedule-induced drinking (Keehn \& Stoyanov, 1983; Patterson \& Boakes, 2012; Staddon \& Ayres, 1975). The manner in which schedule-induced drinking develops indicates that adjunctive behavior is initially reinforced by food occurring in close proximity to licking (e.g., Clark, 1962).

The addition of the lick-food contingency, though it facilitated the development of schedule-induced drinking, did not transform the nature of the behavior. Final temporal location of licking was always in the same position of the interfood interval (i.e., at the beginning of such intervals). This is a strong argument for the preservation of the induced (postprandial, food-related) character of licking (despite the lick-food contingency) and that its association with food occurred even when food was delivered at a distance (a remote consequence within interfood intervals) in relation to the temporal location of licking, in line with previous reports (Lamas \& Pellón, 1995; Moran \& Rudolph, 1980; Pellón \& Blackman, 1987; Pellón \& Pérez-Padilla, 2013) and with the proposal of a recent theory of adjunctive behavior based on the delayed food reinforcement of licking. Specifically, it has been proposed (Killeen \& Pellón, 2013) that adjunctive behavior is maintained by delayed reinforcement, occurring despite the absence of any programmed contingency between the behavior and the consequence, because traces of behaviors of this kind decay at a slow rate, thus maintaining sufficient strength even when the behavior is separated in time from the occurrence of the reinforcer. Furthermore, Pellón and 
Killeen (2015) have recently emphasized that the postfood location of licking is due to its elicitation by food and by competition from other behaviors dominating later in the interfood interval.

The present research therefore shows that drinking can be schedule induced and at the same time maintained (strengthened) by its consequences. If the strengthening aspect is minimized, then the level of responding is reduced. This can be clearly seen in the performance of yoked controls: Their mean licks changed from 200 in the last session of Phase 1 (when no contingency was arranged between their licking and the interfood interval duration), to 400 in the first session of Phase 2, and to over 700 (three- to four-fold greater) during final sessions. It should be noted that, throughout the first phase of the experiment, yoked control animals slightly increased the mean number of licks in correspondence with the shortening of the interfood intervals by the licking of master rats. Despite the optimal conditions of food frequency to develop schedule-induced drinking by the end of the first experimental phase, substantial licking by yoked rats was not clearly manifested until the lick-food contingency was established for this group in the second phase of the experiment. The operant contingency helped to develop scheduleinduced drinking under environmental conditions (food frequency) that, by themselves, did not induce that much licking, and the degree of contingency established between licking and food delivery determined the level of acquisition of scheduleinduced drinking. The present results are in line with previous demonstrations that schedule-induced drinking can be further increased by giving extra food contingent upon licking (Reberg, 1980) or by increasing its associability (Patterson $\&$ Boakes, 2012).

Although not the direct concern of this experiment, magazine entries raise an interesting question that should be considered. In terms of magazine entries, master animals showed the lowest rate, followed by the yoked control group (see Fig. 1, middle panel). The animals with the highest number of entries throughout the experiment were those of the $50 \%$ contingency group. It is interesting to note that, for this group, it took nine sessions to develop schedule-induced drinking and that it reached the response level of master animals at the end of the experiment. However, the rate of magazine entries was the highest of all three groups; very different from the mean values of the master group (more than two-fold at the end of both phases of the experiment). A possible explanation for these results may come from the existent uncertainty about the delivery of the food pellet by the use of a partial reinforcement schedule in Phase 1. The random presentation of yoked and master trials may have led to a constant goal-checking behavior (Boakes, 1979) that was maintained throughout the whole experiment.

As shown in Fig. 4, the distribution of magazine entries for the $50 \%$ contingency group is slightly different from that of the master group. Compared to master subjects, the $50 \%$ contingency animals showed elevated magazine entries from second 15 to second 30 (approximately). These extra entries might have been due to the fact that, during Phase 1, $50 \%$ of the intervals were controlled by master subjects. Partial reinforcement promotes high levels of conditioned response (licking, in the present case; e.g., Goodrich, 1959; Kaye \& Pearce, 1984), and as neither yoked nor master trials were signaled, the $50 \%$ contingency rats may have acquired a constant checking behavior to see whether the pellet had been delivered. This situation led to high levels of magazine entries, as well as drinking, during the second experimental phase.

Magazine entries began with a flat distribution along interfood intervals and then showed a biphasic distribution, with a peak of entries at the start of the intervals (associated with the retrieval of the food pellets from the magazine) and another peak at the end. The first peak is not very interesting from the point of view of the learning mechanisms involved, but the second peak is (see Boakes, Patterson, Kendig, \& Harris, 2015, for similar results with constant FT 30-s schedules), as it can be presumed to compete with drinking (e.g., Aristizabal, Callejas-Aguilera, Ogallar, Pellón, \& Rosas, 2015; but see below). Competition between licking a waterspout and magazine entering is a normal finding in temporal schedules (e.g., Reid \& Dale, 1983), as well as for other operant responses (Dragoi \& Staddon, 1999), and in the present results, it can be seen how distributions of licking (see Fig. 3) and magazine entering (see Fig. 4) inversely overlap, both evolving at about the same rate.

During acquisition (Phase 1), master rats (100\% contingency) licked more than the $50 \%$ contingency animals and even more than yoked control rats ( $0 \%$ contingency). However, the $50 \%$ contingency group was the one that showed the highest level of magazine entries, more than the $0 \%$ contingency rats; thus, the order in which animals engaged in licking cannot be attributed solely to competition from magazine entries (despite the master rats showing the highest licking and the lowest entries; see also Boakes et al., 2015). In general, during Phase 1 , licks increased as sessions progressed but magazine entries did not decrease (see the lack of an effect of sessions for magazine entries in the Results section; see also Kirkpatrick \& Church, 2003). Another result that supports the idea that differences in licking should not be attributed exclusively to response competition from magazine entries is that, during Phase 2, the increases in licking observed for the yoked and $50 \%$ contingency groups did not match their reductions in magazine entries, because although increases in licks were accompanied by decreases in entries for yoked control rats (although no statistically significant reduction in magazine entries was reported), such opposite tendencies were not as clear in the $50 \%$ contingency group. The fact that the $50 \%$ contingency animals showed high rates both of magazine entries and schedule-induced drinking 
indicates that these responses are not functioning as competing responses in the present circumstances, or that their competitiveness cannot account solely for the features of scheduleinduced drinking.

\section{Conclusions}

Schedule-induced polydipsia (aka schedule-induced drinking) has been a matter of concern due to its nature (origin). While some authors have considered it as an example of a different class of behavior (adjunctive), others have argued that it is actually an operant that can be acquired by means of delayed reinforcement. If adjuncts were sensitive to contingencies, there would be no need to consider them as a separate class of behavior. In this experiment, results further indicate that schedule-induced drinking is sensitive to the contingencies (positive) established by the experimenter. The gradation in the development of schedule-induced drinking matched the degree of contingency between licking and food delivery, and in all cases, licking producing hastening in the delivery of food was located at the beginning of interfood intervals, with food being delivered at the end. Schedule-induced drinking appears to be located early in interfood intervals and maintained by food at the end, but these aspects do not constitute reasons to consider it different from conventional operant responding maintained by delayed reward (see Killeen \& Pellón, 2013).

Acknowledgments Research and preparation of the manuscript was supported by Spanish Government research grants PSI2011-29399 and PSI2014-56944-P (Ministerio de Economía y Competitividad: Secretaría de Estado de Investigación, Desarrollo e Innovación) to Ricardo Pellón. Thanks to Jack Marr for the insight into the experimental question (though he might not be aware), and to Alejandro Higuera, Miguel Miguéns, and Peter R. Killeen for comments on previous versions of the manuscript.

\section{References}

Aristizabal, J. A., Callejas-Aguilera, J. E., Ogallar, P. M., Pellón, R., \& Rosas, J. M. (2015). Contextual control of extinguished scheduleinduced drinking and magazine behavior within an ABA renewal design in rats. Psicológica, 36, 337-366.

Baum, W. M. (2012). Rethinking reinforcement: Allocation, induction, and contingency. Journal of the Experimental Analysis of Behavior, 97, 101-124.

Boakes, R. A. (1979). Performance on learning to associate a stimulus with positive reinforcement. In H. Davis \& H. M. B. Hurwitz (Eds.), Operant-Pavlovian interactions (pp. 67-97). Hillsdale, NJ: Erlbaum.

Boakes, R. A., Patterson, A. E., Kendig, M. D., \& Harris, J. A. (2015). Temporal distributions of schedule-induced licks, magazine entries, and lever presses on fixed-and variable-time schedules. Journal of Experimental Psychology: Animal Learning and Cognition, 41, 52-68.
Bolles, R. C. (1961). The interaction of hunger and thirst in the rat. Journal of Comparative and Physiological Psychology, 54, 580-584.

Clark, F. C. (1962). Some observations on the adventitious reinforcement of drinking under food reinforcement. Journal of the Experimental Analysis of Behavior, 5, 61-63.

Dragoi, V., \& Staddon, J. E. R. (1999). The dynamics of operant conditioning. Psychological Review, 106, 20-61.

Falk, J. L. (1961). Production of polydipsia in normal rats by an intermittent food schedule. Science, 133, 195-196.

Falk, J. L. (1966a). The motivational properties of schedule-induced polydipsia. Journal of the Experimental Analysis of Behavior, 9, 19-25.

Falk, J. L. (1966b). Schedule-induced polydipsia as a function of fixed interval length. Journal of the Experimental Analysis of Behavior, 9, 37-39.

Falk, J. L. (1967). Control of schedule-induced polydipsia: Type, size, and spacing of meals. Journal of the Experimental Analysis of Behavior, 10, 199-206.

Falk, J. L. (1969). Conditions producing psychogenic polydipsia in animals. Annals of the New York Academy of Sciences, 157, 589-593.

Falk, J. L. (1971). The nature and determinants of adjunctive behavior. Physiology and Behavior, 6, 577-588.

Flores, P., \& Pellón, R. (1995). Rate-dependency and the rate-decreasing effects of d-amphetamine on schedule-induced drinking. Behavioral Pharmacology, 6, 16-23.

Flory, R. K. (1971). The control of schedule-induced polydipsia: Frequency and magnitude of reinforcement. Learning and Motivation, 2, 215-227.

Goodrich, K. P. (1959). Performance in different segments of an instrumental response chain as a function of reinforcement schedule. Journal of Experimental Psychology, 57, 57-63.

Íbias, J., Pellón, R., \& Sanabria, F. (2015). A microstructural analysis of schedule-induced polydipsia reveals incentive-induced hyperactivity in an animal model of ADHD. Behavioural Brain Research, 278, 417-423.

Job, R. E. S. (1988). Interference and facilitation produced by noncontingent reinforcement in the appetitive situation. Animal Learning and Behavior, 16, 451-460.

Kaye, H., \& Pearce, J. M. (1984). The strength of the orienting response during Pavlovian conditioning. Journal of Experimental Psychology: Animal Behavior Processes, 10, 90-109.

Keehn, J. D., \& Stoyanov, E. (1983). Disruption of adjunctive drinking by lick-dependent delays in feeding. Psychological Record, 33, 391-400.

Killeen, P. R., \& Pellón, R. (2013). Adjunctive behaviors are operants. Learning and Behavior, 41, 1-24.

Kirkpatrick, K., \& Church, R. M. (2003). Tracking the expected time to reinforcement in temporal conditioning procedures. Learning and Behavior, 31, 3-21.

Kissileff, H. R. (1969). Food-associated drinking in the rat. Journal of Comparative and Physiological Psychology, 67, 284-300.

Knutson, J. F., \& Schrader, S. P. (1975). A concurrent assessment of schedule-induced aggression and schedule-induced polydipsia in the rat. Animals Learning and Behavior, 3, 16-20.

Lamas, E., \& Pellón, R. (1995). Food-deprivation effects on punished schedule-induced drinking in rats. Journal of the Experimental Analysis of Behavior, 64, 47-60.

Lamas, E., \& Pellón, R. (1997). Food deprivation and food-delay effects on the development of adjunctive drinking. Physiology and Behavior, 61, 153-158.

López-Crespo, G., Rodríguez, M., Pellón, R., \& Flores, P. (2004). Acquisition of schedule-induced polydipsia by rats in proximity to upcoming food delivery. Learning and Behavior, 32, 491-499.

Mazur, J. E. (1998). Learning and behavior (4th ed.). Upper Saddle River, NJ: Prentice-Hall.

Moran, G., \& Rudolph, R. L. (1980). Some effects of lick-contingent delays on the development of schedule-induced polydipsia. Learning and Motivation, 11, 366-385.

Patterson, A. E., \& Boakes, R. A. (2012). Interval, blocking and marking effects during the development of schedule-induced 
drinking in rats. Journal of Experimental Psychology: Animal Behavior Processes, 38, 303-314.

Pellón, R. (1990). Polidipsia inducida por programa: I. Definición y marco conceptual [Schedule-induced polydipsia: I. Definition and conceptual framework]. Revista de Psicología General y Aplicada, 43, 313-326.

Pellón, R. (2004). La ley del efecto y la conducta innata [The law of effect and innate behavior]. In R. Pellón \& A. Huidobro (Eds.), Inteligencia y aprendizaje [Intelligence and learning] (pp. 89-114). Barcelona: Ariel.

Pellón, R., \& Blackman, D. E. (1987). Punishment of schedule-induced drinking in rats by signaled and unsignaled delays in food presentation. Journal of Experimental Analysis of Behavior, 48, 417-434.

Pellón, R., \& Castilla, J. L. (2000). Punishment of schedule-induced drinking by lick dependent delays in food presented at different frequencies. The Psychological Record, 50, 141-153.

Pellón, R., \& Killeen, P. R. (2015). Responses compete and collaborate, shaping each other's distributions: Commentary on Boakes, Patterson, Kendig, and Harris (2015). Journal of Experimental Psychology: Animal Learning and Cognition, 41, 444-451.
Pellón, R., \& Pérez-Padilla, A. (2013). Response-food delay gradients for lever pressing and schedule-induced licking in rats. Learning and Behavior, 41, 218-227.

Reberg, D. (1980). Reinforcing the occurrence or nonoccurrence of interim drinking. Animal Learning and Behavior, 8, 120-128.

Reid, A. K., \& Dale, R. H. I. (1983). Dynamic effects of food magnitude on interim-terminal interaction. Journal of the Experimental Analysis of Behavior, 39, 135-148.

Roper, T. J. (1981). What is meant by the term "schedule induced" and how general is schedule induction? Animal Learning and Behavior, 9, 433-440.

Staddon, J. E. R., \& Ayres, S. L. (1975). Sequential and temporal properties of behavior induced by a schedule of periodic food delivery. Behavior, 54, 26-49.

Tang, M., Williams, S. L., \& Falk, J. L. (1988). Prior schedule exposure reduces the acquisition of schedule-induced polydipsia. Physiology \& Behavior, 44, 817-820.

Wetherington, C. L. (1982). Is adjunctive behavior a third class of behavior? Neuroscience and Biobehavioral Reviews, 6, 329-350. 